\title{
Hypereosinophilia as a paraneoplastic phenomenon in non-small- cell lung carcinoma
}

\author{
L Nqwata, ${ }^{1}$ MB ChB, FCP (SA); M L Wong, ${ }^{1}$ MB BCh, DCh (SA), FCP (SA), FCCP, FRCP (Lond); \\ R D Mohanlal, ${ }^{2}$ MB ChB, DMH, FCPath (SA), MMed; A B Lakha, ${ }^{3}$ MB ChB, FCP (SA), Cert Clin Haem (SA) \\ ${ }^{1}$ Division of Pulmonology, Chris Hani Baragwanath Academic Hospital; and the Faculty of Health Sciences, University of the Witwatersrand, \\ Johannesburg, South Africa \\ ${ }^{2}$ Department of Anatomical Pathology, School of Pathology, Faculty of Health Sciences, University of the Witwatersrand; and National Health Laboratory Services, \\ Chris Hani Baragwanath Academic Hospital, Johannesburg, South Africa \\ ${ }^{3}$ Division of Clinical Haematology, Chris Hani Baragwanath Academic Hospital; and the Faculty of Health Sciences, University of the Witwatersrand, \\ Johannesburg, South Africa
}

Correspondingauthor:LNqwata(drlamla@yahoo.com)

Hypereosinophilia is a rare paraneoplastic finding in malignant disease, particularly lung cancer. When it occurs, it is usually indicative of metastatic disease. We describe a 52-year-old male patient with paraneoplastic hypereosinophilia associated with primary adenocarcinoma of the right lower lobe and extensive metastatic disease.

S Afr Respir J 2015;21(4):108-109. DOI:10.7196/SARJ.2015.v21i4.38

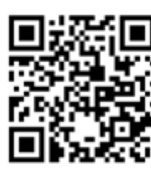

Hypereosinophilia is defined as peripheral blood eosinophilia $\geq 1.5 \times 10^{9} / \mathrm{L}$ on two different occasions at least one month apart, with or without tissue eosinophilia, but without evidence of eosinophilinduced organ damage or dysfunction. ${ }^{[1]}$ Rarely, it may be encountered as a paraneoplastic phenomenon.

\section{Case report}

We report a 52-year-old male patient who presented with a 2month history of cough and significant weight loss. His past

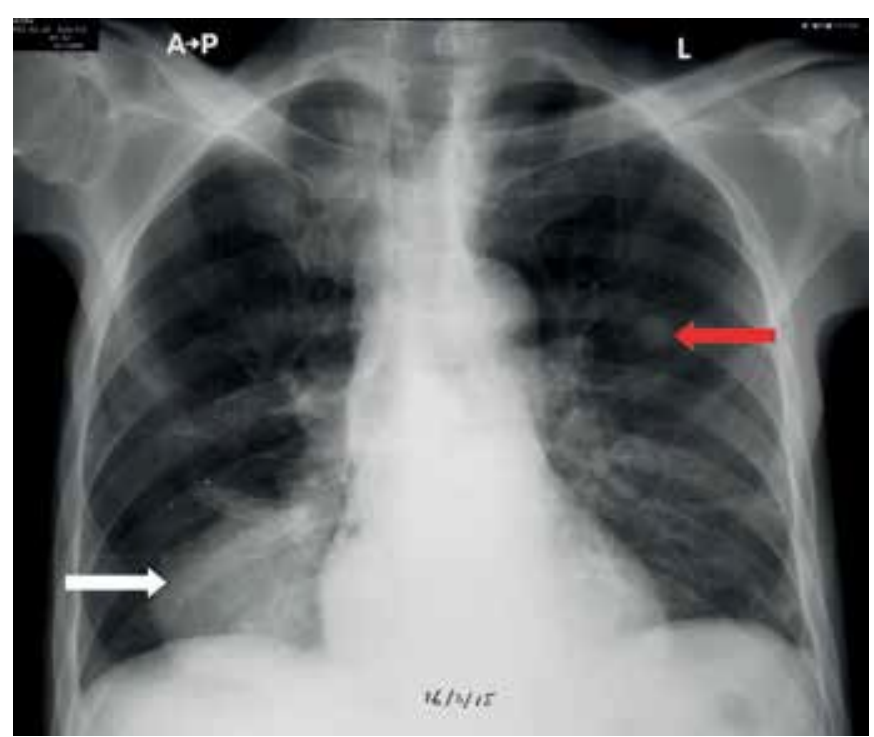

Fig. 1. Chest radiograph showing large mass in the right lower lobe (white arrow) and metastatic pulmonary nodule in the left midzone (red arrow). medical history was unremarkable, except for chronic obstructive pulmonary disease (COPD) and long-standing, well-controlled epilepsy. Physical examination revealed a wasted patient with slow cognition not associated with any focal neurological deficits. Clinical examination revealed decreased breath sounds with dullness to percussion over the right lower lobe, and hepatomegaly extending $8 \mathrm{~cm}$ below the costal margin. Blood investigations showed marked elevation of his white cell count (peak level $114.18 \times 10^{9} / \mathrm{L}$ ) with an absolute eosinophilia ranging from 29.81 to $82.33 \times 10^{9} / \mathrm{L}$ during the course of his admission. The bone marrow trephine confi med marked eosinophilia, with no malignant infiltrate or evidence of clonal eosinophilic proliferation (absence of the FIP1L1/PDGFRA fusion gene on fluorescence in situ hybridisation (FISH) analysis). The chest radiograph showed a well-circumscribed lesion in the

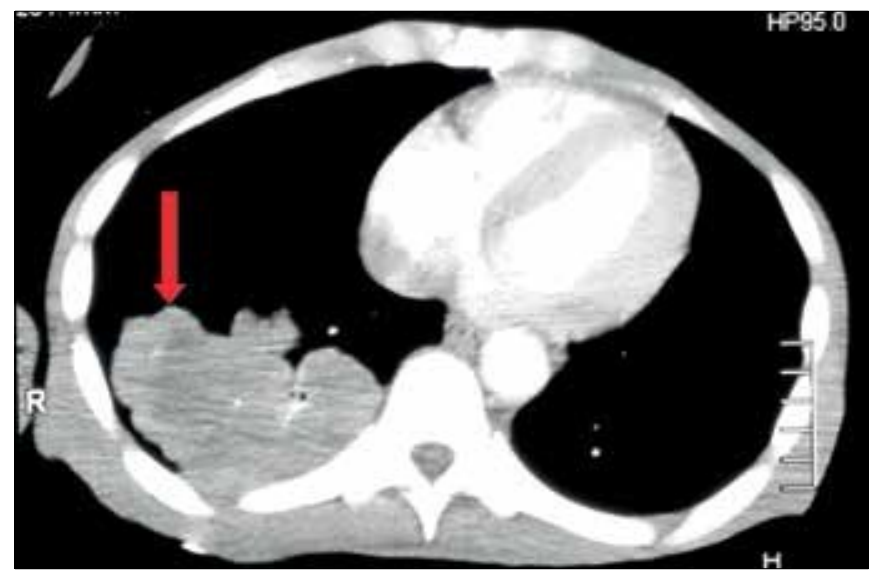

Fig. 2. CT scan demonstrating lobulated right lower lobe mass (red arrow). 
right lower lobe with a pulmonary nodule in the left upper lobe (Fig. 1). Computed tomography demonstrated a right lower lobe mass measuring $9.1 \times 6.0 \times 6.7 \mathrm{~cm}$ (Fig. 2), bilateral pulmonary nodules, multiple liver lesions and a left adrenal mass. A core biopsy of the lung mass revealed nests of tumour cells with abundant eosinophilic cytoplasm and extensive tumour necrosis. No keratinisation or well-formed glands were noted. A panel of immunohistochemical stains was positive for CK7, MOC31 and thyroid transcription factor (TTF-1) in the malignant cells. Other stains performed, including p63, calretinin and WT1, excluded mesothelioma and squamous cell carcinoma. A final diagnosis of poorly differentiated adenocarcinoma of the lung was made.

High-dose corticosteroids and hydroxyurea were administered to the patient, with only modest reduction in the eosinophilia. He was referred to the palliative care team and died 47 days after admission.

\section{Discussion}

Paraneoplastic hypereosinophilia is rare. It has been described with many solid tumours including thyroid, breast, genitourinary, gastrointestinal, hepatocellular and in both non-small- and small-cell lung carcinoma. ${ }^{[2]}$

Several studies suggest that local production of cytokines (GMCSF, IL-3, and IL-5) by tumour cells is the most likely mechanism, particularly IL-5, which is considered to be the most important eosinophilopoetin..$^{[3-5]}$ These cytokines are involved in eosinophil development and maturation, with predominantly IL-5 mobilising eosinophils from bone marrow into the blood.
Management of eosinophilia is best achieved by tumour reduction (surgery and/or chemotherapy). However, case reports suggest that this is not always feasible, as these patients usually have extensive metastases and poor outcomes. ${ }^{[3-5]}$

\section{Conclusion}

Our case report illustrates paraneoplastic hypereosinophilia, a rare manifestation in primary lung cancer. As in other case reports, ${ }^{[3-5]}$ this is associated with a poor prognosis.

\section{Learning points:}

- Hypereosinophilia may occur as a paraneoplastic phenomenon in primary lung cancer.

- Paraneoplastic hypereosinophilia portends a poor prognosis.

\section{References}

1. Valent P, Klion AD, Horny HP, et al. Contemporary proposal on criteria and classification of eosinophilic disorders and related syndromes. J Allergy Clin Immunol 2012;130(3):607-612. [http://dx.doi.org/10.1016/j.jaci.2012.02.019]

2. Samoszuk M. Eosinophils and human cancer. Histol Histopathol 1997;12(3):807-812

3. Venkatesan R, Salam A, Alawin I, Willis M. Non-small cell lung cancer and elevated eosinophil count: A case report and literature review. Cancer Treatment Communications 2015;4:55-58. [http://dx.doi.org/10.1016/j.ctrc.2015.05.002]

4. Pandit R, Scholnik A, Wulfekuhler L, et al. Non-small cell lung cancer associated with excessive eosinophilia and secretion of interleukin-5 as a paraneoplastic syndrome. Am J Hematol 2007;82(3):234-237. [http://dx.doi.org/10.1002/ajh.20789]

5. Verstraeten AS, de Weerdt A, van den Eynden G, et al. Excessive eosinophilia as paraneoplastic syndrome in a patient with non-small-cell lung carcinoma: A case report and review of the literature. Acta Clin Belg 2011;66(4):293-297. [http://dx.doi. org/10.1179/ACB.66.4.2062571] 